\title{
Excessive Right Subdiaphragmatic Fat: False Radiologic Pneumoperitoneum
}

\author{
Dong Hun Kim¹, Jung Ho Yun ${ }^{2}$ \\ ${ }^{1}$ Department of Surgery, ${ }^{2}$ Department of Neurosurgery, Dankook University Hospital, Cheonan, Korea
}

Excessive fat in the right subdiaphragmatic space may simulate radiological pneumoperitoneum. We report a trauma patient with excessive right subdiaphragmatic fat who was examined with plain chest radiography and abdominal computed tomography $(\mathrm{CT})$. Chest radiography showed a radiolucency separating the liver and right hemidiaphragm. The subphrenic fat pad was substantiated by CT.

Key Words: Diaphragm; Abdominal Fat; Pneumoperitoneum

(Trauma Image Proced 2016(1):10-11)

\section{CASE}

A 46-year-old man was admitted to the emergency department with an injury from falling from a 3-m height. The patient presented with transient hypotension and alert mentality. The physical examination revealed multiple head injuries in addition to multiple rib fractures with scanty amount of hemothorax. He underwent an emergency craniectomy for his increasing epidural hemorrhage and compound depressed skull fracture. Supine chest radiography showed a radiolucency separating the liver and right hemidiaphragm (Fig. 1.), which might be mistaken for a pneumoperitoneum. Physical examination revealed a soft abdomen and no peritoneal irritation sign. Focused assessment with sonography for trauma showed no intraabdominal fluid collection. Abdominal computed tomography (CT) demonstrated no evidence of intraabdominal organ injury, and the right subdiaphra-

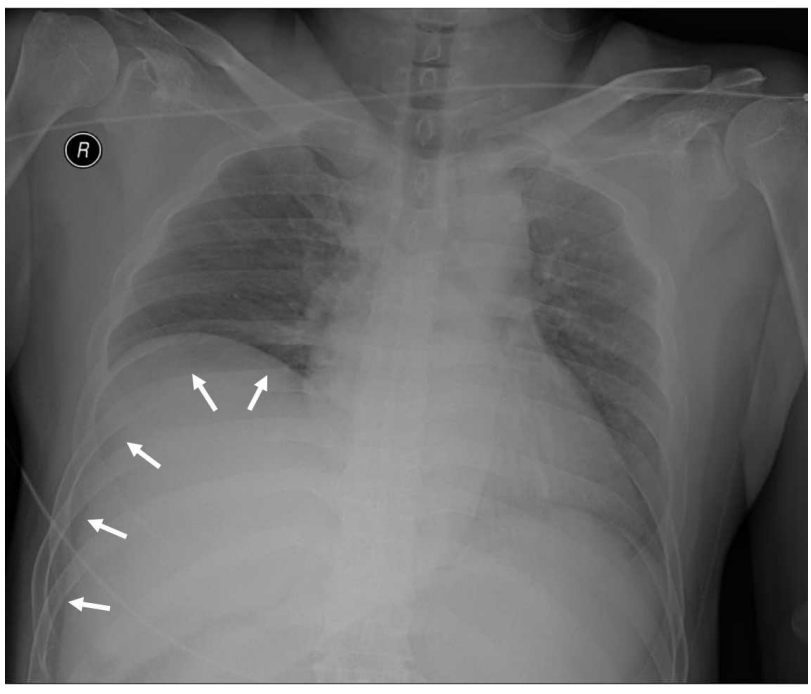

Fig. 1. Supine chest radiography shows a radiolucent lesion separating the liver and right hemidiaphragm (white arrows).

gmatic lesion on chest radiography corresponded to an excessive fat pad (Fig. 2.).

Received: April 24, 2016 Revised: May 3, 2016 Accepted: June 12, 2016

Correspondence to: Dong Hun Kim, Trauma Center, Department of Surgery, Dankook University Hospital, Anseo-dong, Dongnam-gu, Cheonan-si, Chungcheongnam-do, Korea

Tel: 82-41-550-7119, Fax: 82-41-550-0039, E-mail: saint7331@gmail.com

Copyright (c) 2016 Korean Association for Research, Procedures and Education on Trauma. All rights reserved.

@This is an open-access article distributed under the terms of the Creative Commons Attribution Non-Commercial License (http://creativecommons.org/ licenses/by-nc/4.0) which permits unrestricted noncommercial use, distribution, and reproduction in any medium, provided the original work is properly cited 

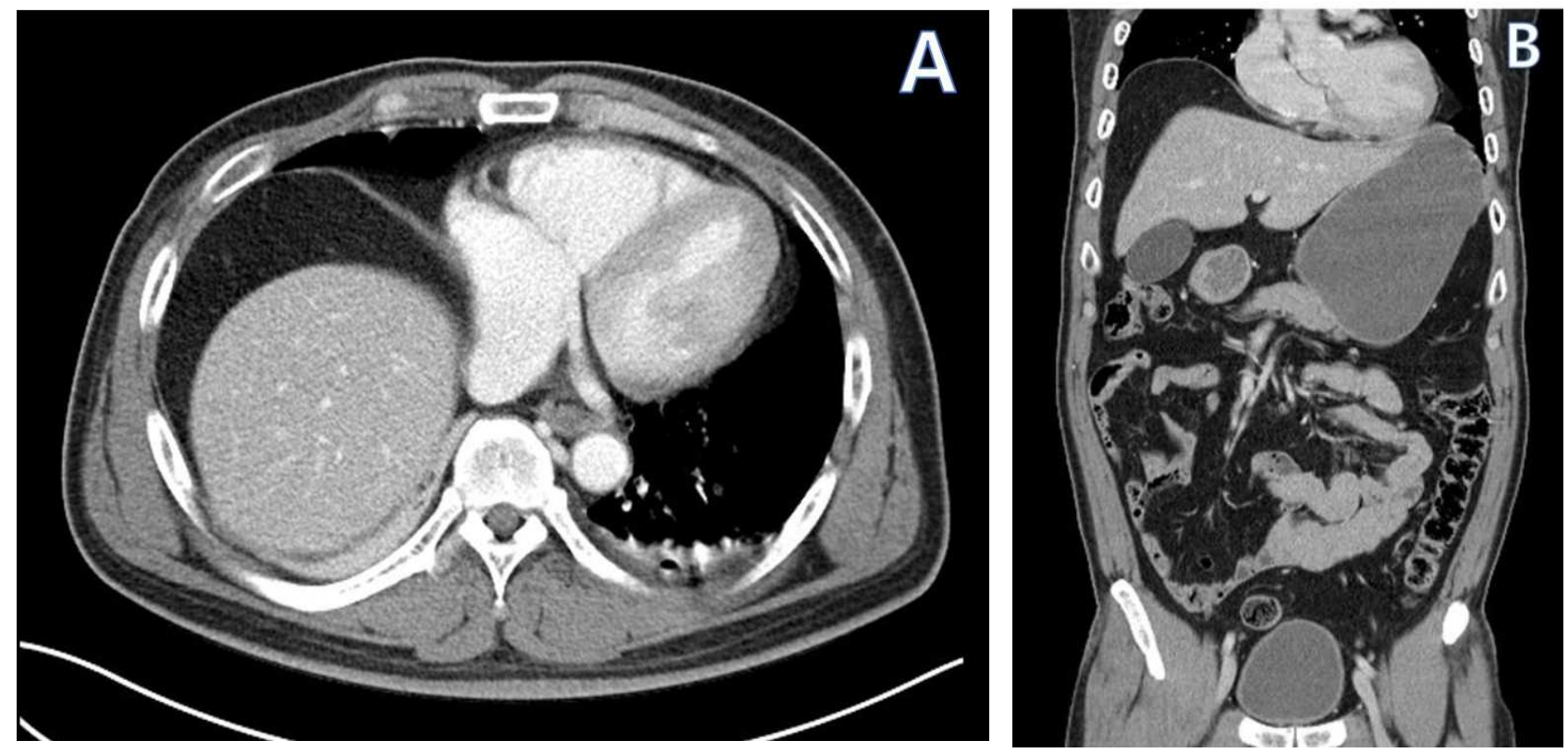

Fig. 2. Abdominal computed tomography shows an intraperitoneal fat pad localized between the diaphragm and liver. (A) Axial view (B) coronal view.

\section{DISCUSSION}

The term pseudopneumoperitoneum was used by Moknohisky for the appearance of subphrenic air due to a fat pad or an irregular leaf of the diaphragm (1). Furthermore, pathological processes such as Chilaiditi syndrome, curvilinear pulmonary collapse, ascites, subphrenic abscess, and subpulmonary pneumothorax can cause pseudopneumoperitoneum $(1,2)$. Subphrenic fat can be mistaken for intraperitoneal air even in normal patients, especially on chest radiography $(1,2)$. CT is the best imaging modality for discriminating this state. The right subdiaphragmatic fat pad might be arising from the greater omentum or abnormal fat deposition resulting from corticosteroid use (1-3).

\section{CONFLICT OF INTEREST}

No potential conflict of interest relevant to this article was reported.

\section{REFERENCES}

1. Mokrohisky JF. Pseudopneumoperitoneum; simulated free air in the peritoneal cavity. The American journal of roentgenology, radium therapy, and nuclear medicine. 1958; 79(2):293-300.

2. Rao KG, Woodlief RM. Excessive right subdiaphragmatic fat: a potential diagnostic pitfall. Radiology. 1981;138(1): 15-8.

3. Martinez LO, Raskin MM. Fat under diaphragm simulating pneumoperitoneum. The British journal of radiology. 1974; 47(558):308-9. 